\title{
LE SAUMON POLONAIS
}

\author{
par R. BACHELIER, \\ Ingénieur principal des Laux et Forêts.
}

II. le docteur Derriox ayant qualifié de "mystérieux" le Saumon de Pologne, je me permets de rendre compte ci-dessous de ce que j'ai appris de celui-ci et de son concurrent la Truite du Dunajec, lors d'une mission que le Conseil Supérieur de la Pèche mavait confiée dans ce pays en 1960.

Pendant plusieurs années, de 1954 à 1959 inclus, il m'avait été possible de me procurer des œufs de Saumons de Pologne en échange de civelles ; mais à vrai dire de moins en moins, et en 1960 plus du tout, alors qu'il m'était offert des œufs de Truites du Dunajec en quantité illimitée.

Quand on sait combien sont identiques les œufs des uns et des autres, et combien est faible la différence morphologique entre les Saumons polonais et les Truites du Dunajec qui, les uns comme les autres, pèsent normalement $7-8 \mathrm{~kg}$, je ne pouvais que m'incliner devant l'honnêteté des pisciculteurs polonais.

Cependant, depuis 6 ans que je faisais enfouir une centaine de milliers d'œufs de Saumons polonais et plusieurs centaines de milliers d'œufs de Truites du Dunajec dans divers fleuves de France, il n'avait été constaté que quelques remontées de Saumons (Gartempe, Blavet) et aucune de grosse Truite de mer.

La quasi-totalité du réseau fluvial de la Pologne est encore remontée par des salmonides anadromes, comme celui de la France... jadis !... Mais avec cette différence : c'est que tous ces salmonides y pèsent au minimum 3 ou $4 \mathrm{~kg}$ et leur grande majorité 7 ou $8 \mathrm{~kg}$ alors qu'en France, il y a des Truites de mer qui ne pèsent même pas le kilogramme.

La raison de cette différence est que tous ces salmonides anadromes polonais passent leur vie marine dans le mer Baltique qui, comme chacun sait, est peu profonde, peu salée et fort riche en nourriture.

\section{LES SALMONIDES ANADROMES EN MER BALTIQUE}

La preuve est donnée que les salmonides anadromes polonais ne sortent pas de la mer Baltique par le fait que jamais un smolt ayant été bagué en Pologne n'a été repris en mer du Nord. Il en a d'ailleurs été de même pour les smolts ayant été bagués en Suède sur la côte Baltique (Kmit Dahl et Seven Somme, 1935). 
A vrai dire, l'absence de migrations de Saumons à travers le Kategatt n'est pas surprenante quand on sait le peu d'ancienneté de ce détroit et l'effort de pêche qui y est pratiqué.

\section{RECENSEMENT DES CAPTLRES DE SALMONIDES \\ DANS LA BALTIQLE, EN TONNES}

(publication de M. Feliks ChrzaN)

\begin{tabular}{|c|c|c|c|c|c|c|c|c|}
\hline \multirow{2}{*}{ AnNées } & \multirow{2}{*}{$\mid \begin{array}{c}\text { Salmo } \\
\text { Thutta }\end{array}$} & \multicolumn{7}{|c|}{ SALMo SALAR } \\
\hline & & Pologne & Suèrle & $\begin{array}{l}\text { Dane- } \\
\text { mark }\end{array}$ & $\begin{array}{l}\text { Fin- } \\
\text { lande }\end{array}$ & $\begin{array}{c}\text { Alle- } \\
\text { magne }\end{array}$ & C.R.S.S. & TotAL \\
\hline 1946 & 66 & 213 & 1.474 & 475 & 475 & $\ldots$ & 一 & 2.637 \\
\hline 1947 & 64 & 416 & 1.218 & 630 & 395 & - & 161 & 2.820 \\
\hline 1948 & 60 & 346 & 1.248 & 851 & 289 & 6 & 280 & 3.020 \\
\hline 1949 & 46 & 240 & 1.102 & 843 & 260 & 59 & 185 & 2.689 \\
\hline 1950 & 54 & 312 & 1.404 & 1.317 & 400 & 183 & 155 & 3.771 \\
\hline 1951 & 46 & 82 & 1.077 & 1.096 & 352 & 136 & 151 & 2.894 \\
\hline 1952 & 24 & 43 & 790 & 1.328 & 383 & 144 & 149 & 2.837 \\
\hline $1953 \ldots$ & 47 & 24 & 422 & 753 & 350 & 75 & 117 & 1.741 \\
\hline 1954 & 102 & 43 & 484 & 962 & 300 & 116 & 96 & 2.001 \\
\hline 1955. & 26 & 16 & 296 & 609 & 300 & 158 & 110 & 1.489 \\
\hline
\end{tabular}

Dans cette publication M. Feliks Chrzan a signalé deux faits qui m'ont été confirmés à Gdansk (Dantzig) :

$1^{\circ}$ La plupart des captures polonaises enregistrées sur le tableau ci-dessus furent faites à la ligne; avant que ce mode de pêche ne fut pratiqué, c'est-à-dire avant 1945, les captures de salmonides étaient beaucoup plus rares ;

$2^{\circ}$ Les Truites de mer ne sont pèchées que dans les estuaires et dans les eaux littorales peu profondes (moins de 10 mètres), alors qu'au contraire les Saumons ne le sont qu'en pleine mer.

Cette localisation de la vie marine des Truites de mer m'a donné l'explication de l'échec apparent de l'introduction des Truites du Dunajec dans les fleuves côtiers français, alors que, pourtant, nombreux de ces fleuves étaient de nouveau fréquentés par des Truites de mer qui avaient disparu depuis des lustres.

Ne disposant pas des riches "paturages" des eaux littorales de la Baltique, les Truites de mer issues des œufs de Truites du Dunajec grossissaient beaucoup moins vite sur les côtes françaises et n'avaient donc donné lieu qu'à des remontées de poissons de 1 à $3 \mathrm{~kg}$. 


\section{Sarumon}

D'après ce recensement des captures en mer Baltique, la régression du Saumon polonais était donc bien réelle, et il se pouvait d'ailleurs que les efforts de péche conjugués de tous les pays riverains de cette mer n'y aient pas été étrangers.

M. Felicks Chrzan signale encore que la dimension moyenne des Saumons capturés par les Polonais était de $91,3 \mathrm{~cm}$ en $1945-1946,96,3 \mathrm{~cm}$ en $1946-1947,100,3 \mathrm{~cm}$ en $1947-1948$, puis ensuite qu'elle est tombée à $77 \mathrm{~cm}$ et correspondait à un âge de $4+$ au lieu de $5+$.

Par la scalimétrie, il leur avait trouvé la vitesse de croissance suivante :

- 1re année : $22,5 \mathrm{~cm}$ pour $510 \mathrm{~g}$;

- $2^{\mathrm{e}}$ année : $29,7 \mathrm{~cm}$ pour $2.750 \mathrm{~g}$;

- $3^{\mathrm{e}}$ année : $25,7 \mathrm{~cm}$ pour $5.600 \mathrm{~g}$;

- $4^{\mathrm{e}}$ année $: 7,5$ à $13,5 \mathrm{~cm}$ pour 2.800 à $4.300 \mathrm{~g}$.

\section{Truite de mer}

La moyenne annuelle des captures de Truites de mer s'est élevée à 60 tonnes avec un maximum de 102 tonnes en 1954. De nombreuses études scalimétriques ont permis de connaître leurs accroissements annuels, qui sont légèrement plus grands pour les Truites de la Vistule (Dunajec) que pour les Truites des petits fleuves côtiers polonais.

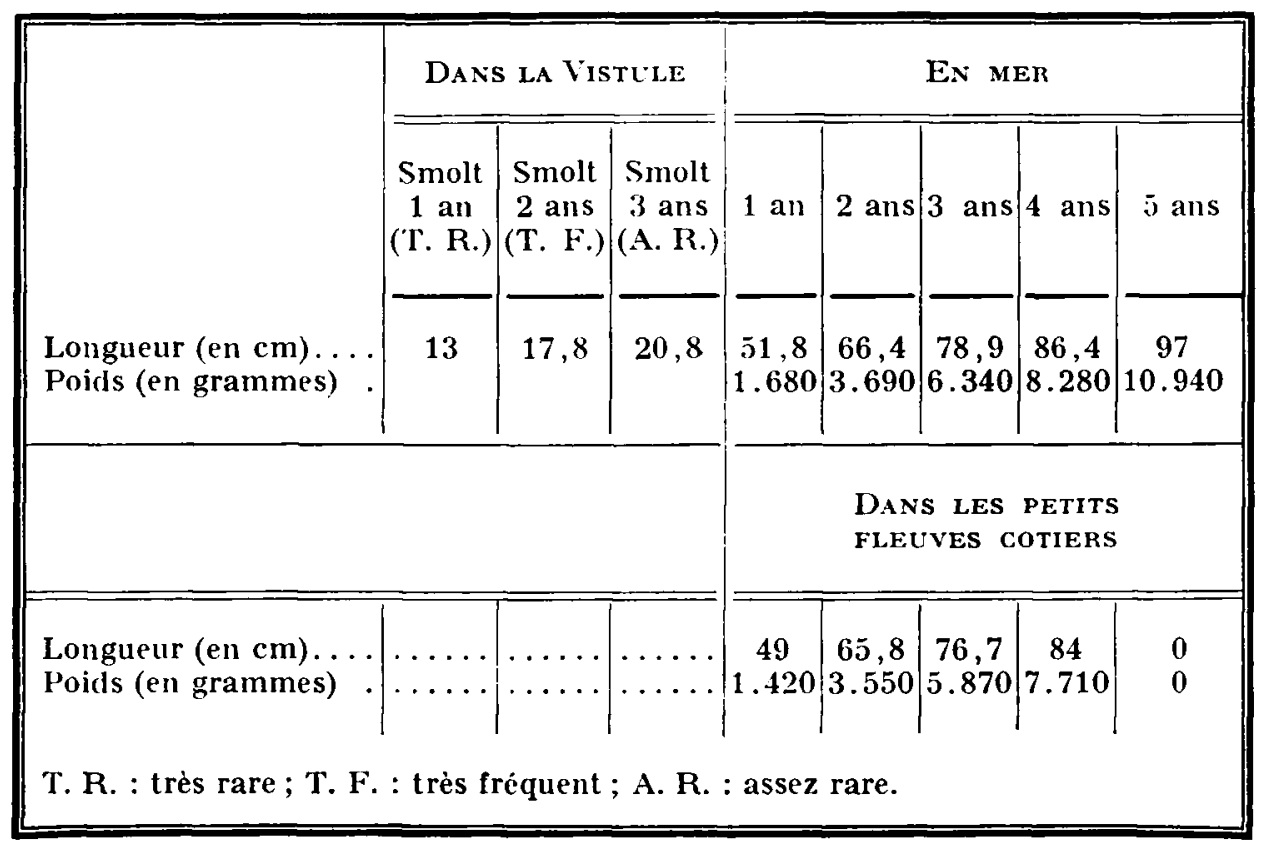


La plupart remonte frayer après 2 ans de mer et $36 \%$ après 3 ans. Il y a deux périodes de remontée en rivière, une à la fin de l'automne et l'autre à la fin de l'hiver.

\section{LES REMONTÉES DES SALMONIDES ANADROMES}

\section{Régression des Saumons}

Sauf quelques exceptions, les Saumons, quand il y en avait encore... remontaient exclusivement dans les affluents supérieurs de la Vistule, la Sola et la Skawa, dans les Karpathes, les autres afluents de laVistule, tel le fameux Dunajec (affluent droit prenant sa source dans le Tatras) étant remontés par de grosses Truites de mer qui pèsent de 7 à $10 \mathrm{~kg}$, et les petits fleuves côtiers par des Truites de mer de 4 à $8 \mathrm{~kg}$.

Cette différence de taille correspond d'ailleurs à une nécessité physiologique puisque les Truites du Dunajec doivent remonter à $800 \mathrm{~km}$, si ce n'est même à $900 \mathrm{~km}$ de la Baltique pour frayer, tout comme les Saumons.

Mais pourquoi les Saumons de la Sola et de la Skawa ont-ils été remplacés par des Truites de mer type Dunajec?

Le Dr ZarNecki, professeur à l'Université de Cracovie, estime que ce remplacement s'explique facilement par une modification du milieu.

Effectivement, de par la répartition de leurs habitats respectifs originels, la Truite de mer se contente d'eaux moins froides que le Saumon. D'ailleurs, il avait été constaté que la dimension moyenne des smolts de Saumons était de $12 \mathrm{~cm}$, tandis que celle de ceux de Truites de mer était de $18 \mathrm{~cm}$.

Le réchauffement climatique 1945-1955, qui en France, avait souvent fait régresser la Truite devant le Brochet ou le Chevesne, peut avoir eu le même résultat pour le Saumon vis-à-vis de la Truite de mer en Pologne.

En outre, sans avoir été rapide avant 1960, il est évident qu'une certaine industrie s'était développée depuis la guerre dans les bassins de la Sola et de la Skawa. En ce qui concerne la Sola notamment, il a été créé dans son bassin supérieur une retenue hydroélectrique de 500 ha, qui a pu, à elle seule, modifier suffisamment le milieu à son aval pour que celui-ci soit devenu plus propice à la Truite de mer qu'au Saumon.

A moins qu'il ne se soit passé pour ces deux rivières des Karpathes ce qui s'est passé pour le fameux Dunajec... et pour la majorité des rivières françaises...

\section{Régression des Truites du Dunajec}

Un barrage-usine de 32 mètres de hauteur fut construit sur le Dunajec en 1940-1941 à Rosnov (altitude de 250 mètres seulement, c'est-à-dire à l'extrémité aval de la zone des frayères de la Truite de mer). Ce barrage 
fut pourvu d'une échelle à poissons monumentale à bassins successifs qui était bien située et qui, d'ailleurs, permit encore une remontée de Truites de mer spectaculaire en 1948.

A quelques kilomètres à son aval, à Czchow, il fut construit en 1949 un barrage de compensation de 9 mètres de hauteur, lui aussi pourvu d'une échelle à poissons qui permet toujours des remontées de Truites de mer. Cependant, celles-ci sont devenues insignifiantes.

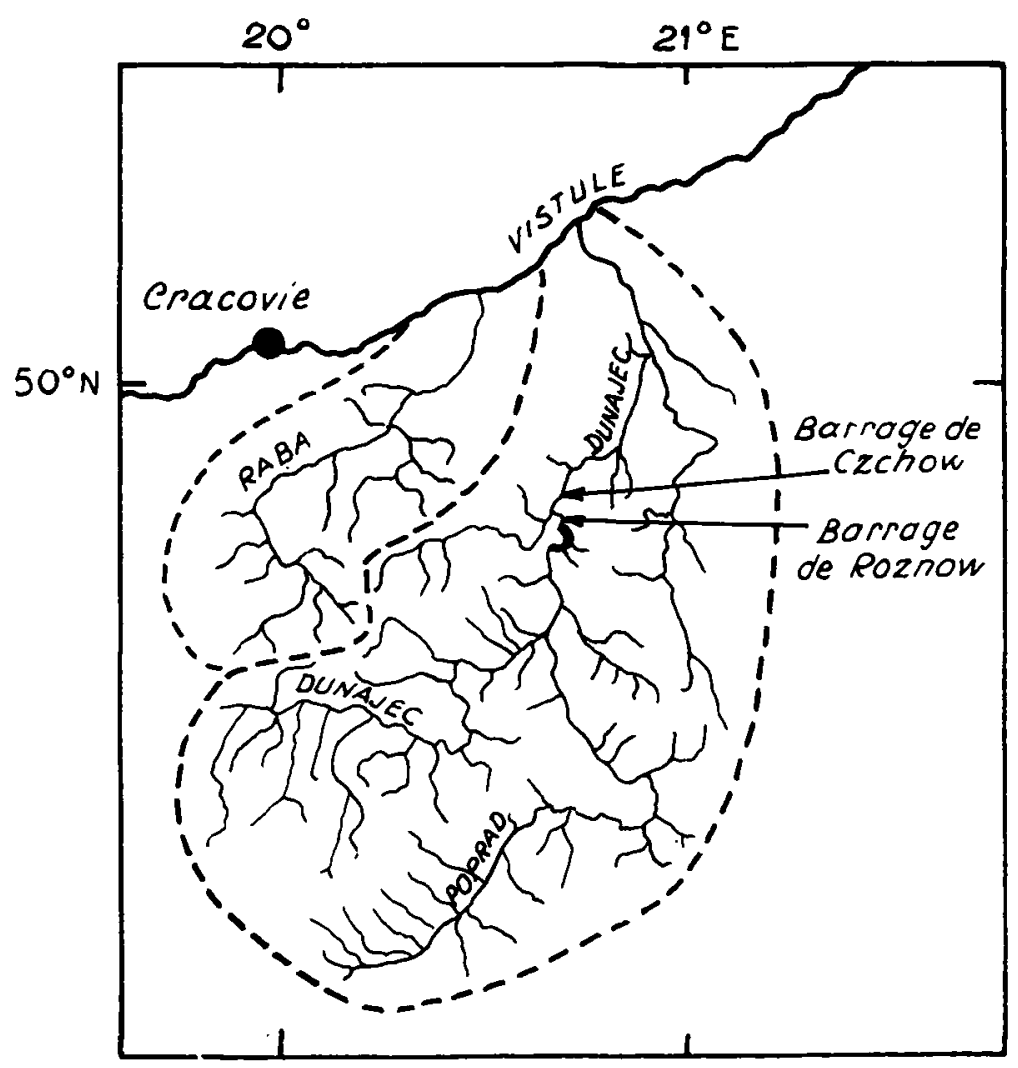

Les deux barrages de la riviere DUNAJEC.

La ligne pointillée délimite les bassins des rivières Raba $\left(1.518 \mathrm{~km}^{2}\right)$ et Dunajec $\left(6.823 \mathrm{~km}^{2}\right)$.

Il y aurait encore une trentaine de frayères fréquentées entre les deux barrages, mais plus aucune à l'amont du barrage de Rosnov.

Plusieurs explications sont possibles, notamment une simple carence dans le fonctionnement de l'échelle à poissons en raison des variations de la hauteur d'eau dans la retenue ; en effet, pour que celle-ci soit correcte, il faut constamment manœuvrer les vannes permettant l'alimentation de l'échelle à partir du bassin supérieur se trouvant au niveau de la retenue. 
De ce fait, la reproduction des Truites de mer dans le Dunajec est limitée maintenant à la capacité de production des frayères situées à l'aval du barrage de Rosnov, c'est-à-dire très à l'aval de la zone à Truites.

\section{Progression des remontées de Truite de mer dans la Raba}

Cet affluent droit de la Vistule, situé entre le Dunajec et la Skawa, était fréquenté avant guerre par quelques Saumons, par des Truites de mer identiques à celles du Dunajec, et surtout par des Hotus.

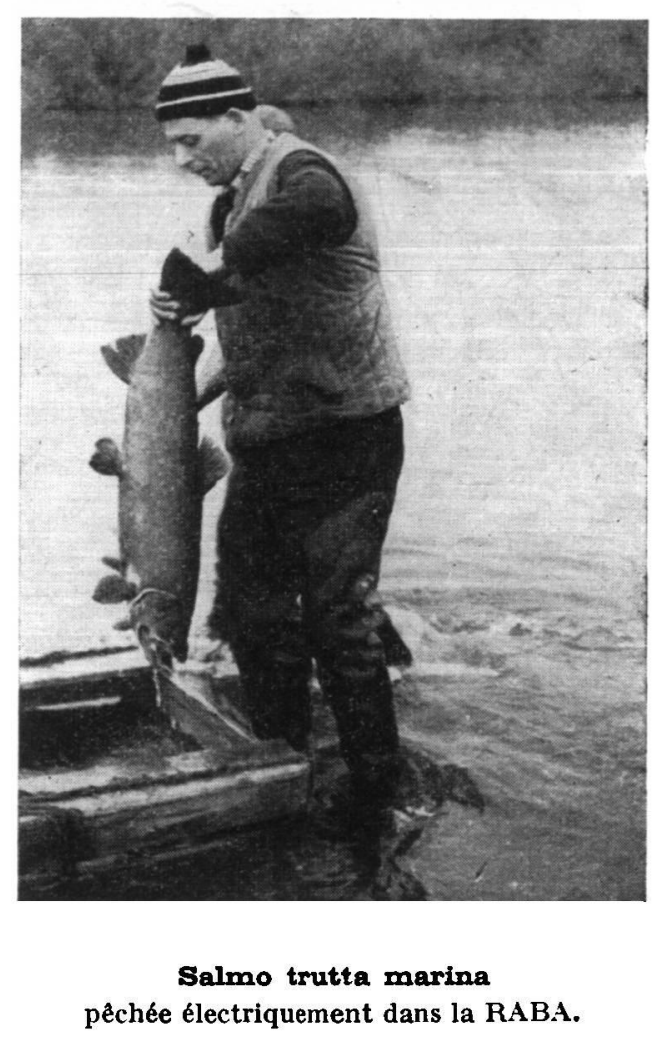

Sa proximité de la pisciculture d'État de Mydlniki, dirigée par Mlle \SKRochorska, permit à celle-ci d'entreprendre la production de smolts de Truites de mer de la Raba par élevage semi-naturel dès 1933. Sur 12.000 smolts déversés bagués dans la Raba de 1951 à 1958, 12 furent repris dans la Vistule dans le mois ayant suivi le déversement, 300 dans le golfe de Dantzig (moitié avant 2 mois, moitié après 2 mois), 33 en Baltique et 55 remontant la Vistule après un séjour en mer de 17 mois en moyenne. Cependant que 900 furent repris dans la Raba qu'ils n'avaient jamais quittée. 


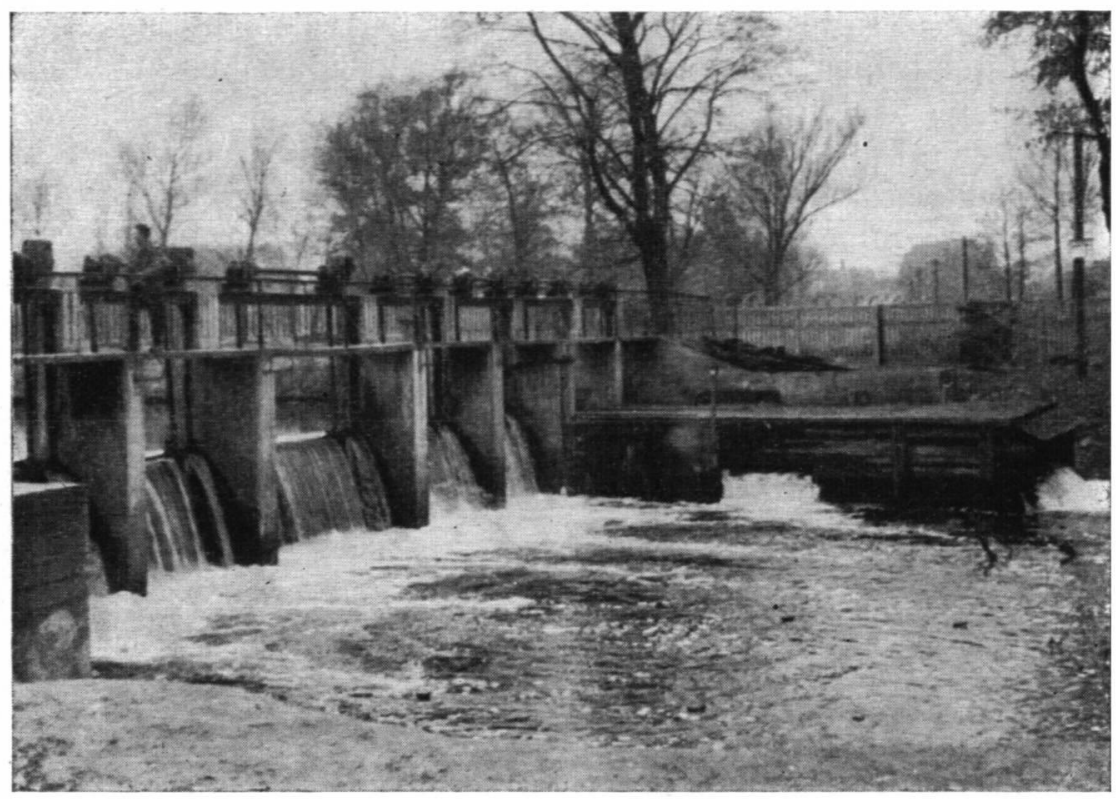

Piege a Truites de mer dans un petit fleuve cótier, a SLUPSK.

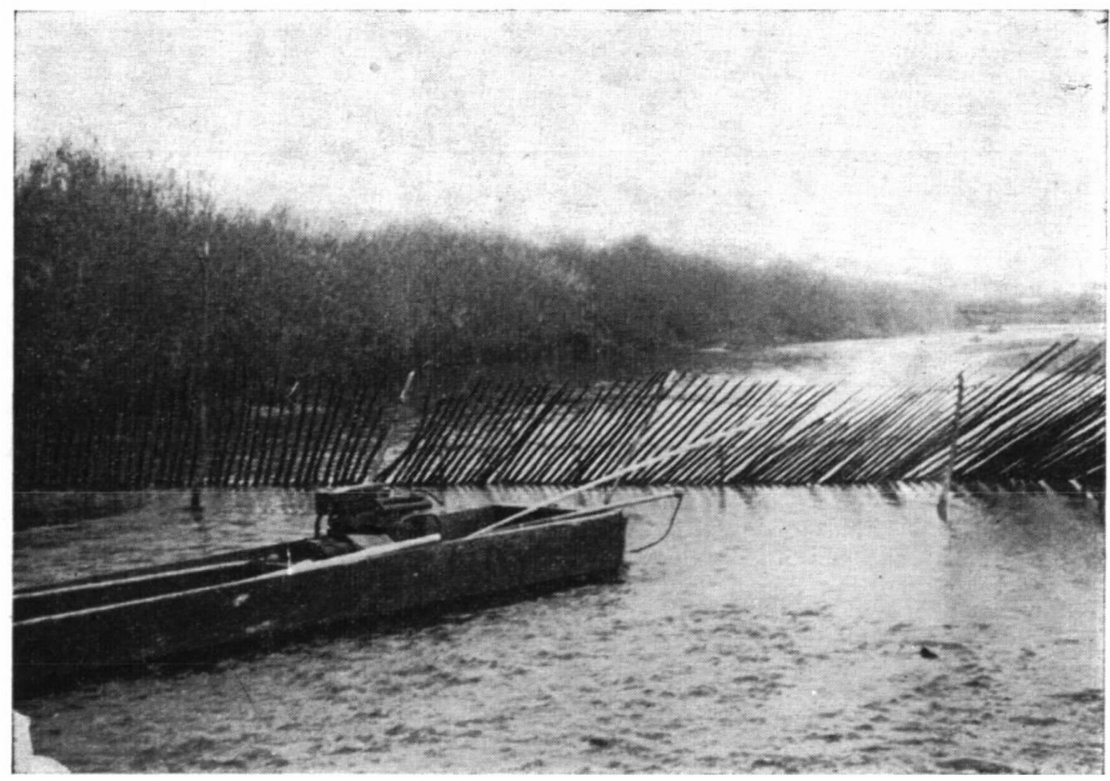

Barrago compartimentant la RABA avant capture des géniteurs de Salmo trutta au moyen de l'agrégat chargé sur le bateau. 
Ce long effort de Mlle Skrochorska, qui a confirmé que le caractère migratoire des Truites de mer n'était que partiellement héréditaire, a permis de quadrupler au moins le peuplement de la Raba en Truites de mer.

Mais, à vrai dire, cet enrichissement remarquable, n'a pas été dû au seul alevinage car, simultanément, la Fédération des pêcheurs à la ligne a engagé une équipe de pêcheurs professionnels qui capturent les hotus à longueur d'année, si bien que ceux-ci ont été littéralement décimés. Aujourd'hui, avec ses Truites de mer de 7 à $9 \mathrm{~kg}$, la Raba a une valeur halieutique égale aux meilleures rivières à Saumons. Mais le Saumon polonais, s'il n'est pas mystérieux, n'est probablement plus qu'un souvenir. 